\title{
Expression of amyloid precursor-like protein 2 (APLP2) in glioblastoma is associated with patient prognosis
}

\author{
Yihong Chen ${ }^{1,2^{*}}$, Hongxiang Wang ${ }^{1^{*}}$, Cong $\operatorname{Tan}^{3}$, Yong Yan ${ }^{1}$, Junyan Shen ${ }^{4}$, Qilin Huang ${ }^{1}$, Tao Xu ${ }^{1}$, Junting Lin $^{5}$, \\ Juxiang Chen ${ }^{1}$ \\ ${ }^{1}$ Department of Neurosurgery, Changzheng Hospital, Second Military Medical University, Shanghai, ${ }^{2}$ Department of Cardiology, \\ Changzheng Hospital, Second Military Medical University, Shanghai, ${ }^{3}$ Department of Pathology, Shanghai Cancer Center, \\ Fudan University, Shanghai, ${ }^{4}$ Department of Regenerative Medicine, Tongji University School of Medicine, Shanghai, \\ ${ }^{5}$ Taizhou Cancer Hospital, The Second People's Hospital of Wenling City, Zhejiang, China \\ ${ }^{*}$ The authors have contributed equally to the study and should both be considered first authors.
}

\begin{abstract}
The purpose of this study was to investigate the expression status of amyloid precursor-like protein 2 (APLP2) and its clinical relevance in patients with glioblastoma. The publically available database Project Betastasis involving Repository for Molecular Brain Neoplasia Data (REMBRANDT) and The Cancer Genome Atlas (TCGA) was first utilized to analyze the expression and prognostic potential of APLP2 in glioblastoma. Compared with normal controls, the glioblastoma group from each dataset showed no significant difference of APLP2 expression ( $p>0.05)$. However, when connected to glioblastoma patient's prognosis, a high APLP2 expression was found to be associated with short overall survival in REMBRANDT cases $(p=0.0323)$ but not the TCGA group $(p=0.0578)$. Consistently, APLP2 expression detected by immunohistochemistry in our cohort revealed an undifferentiated expression pattern between glioblastoma $(n=114)$ and normal brain $(n=16)(p=0.265)$ and among all grade gliomas. Furthermore, univariate and multivariate analyses identified a high APLP2 expression as an independent risk factor for overall survival (hazard ratio $=1.537, p=0.041$ ) and progression-free survival (hazard ratio $=1.783, p=0.037$ ) of glioblastoma patients. In conclusion, the expression of APLP2 might correlate with tumor development and be a prognostic factor for patients with glioblastoma.
\end{abstract}

Key words: APLP2, glioblastoma, prognosis, project betastasis.

\section{Introduction}

Glioblastoma is one of the most common primary tumors in the central nervous system and the most malignant tumor among gliomas [31]. For years, median survival rates for glioblastoma patients have hardly improved with conventional therapeutic strategies, including surgery, radiation, and chemotherapy [20]. Recently, a significant endeavor in investigation of cancer-specific molecular networks

Communicating authors

Juxiang Chen, Department of Neurosurgery, Changzheng Hospital, 415 Fengyang Road, Shanghai 200003, China,

fax: +86-21-63586116, e-mail: juxiangchen@smmu.edu.cn; Junting Lin, Taizhou Cancer Hospital, 22 Jiangkang Road, Wenling 317502 , Zhejiang, China, fax: +86-0576-86590019, e-mail: ljt609@sohu.com 
has drastically advanced our understanding of glioblastoma pathophysiology, such as three core pathways, receptor tyrosine kinase (RTK)/RAS/phosphatidylinositol 3-kinase (PI3K), p53 and retinoblastoma protein $(\mathrm{RB})$, underlying gliomagenesis found by The Cancer Genome Atlas (TCGA) [7]. Identification of functional therapeutic targets that participate in the regulation of glioblastoma development and molecular factors associated with patient prognosis may provide new opportunities for treating this lethal cancer and further selecting individual patients for a specific therapeutic modality.

Amyloid precursor-like protein 2 (APLP2) is a type I transmembrane protein and a member of the amyloid precursor protein family, which is highly conserved and ubiquitously expressed $[10,30]$. As reported previously, APLP2 has biochemical functions related to cell migration, signaling, adhesion, proliferation, and healing $[9,13,17,32]$. Recently, dysregulation of APLP2 has been found in multiple cancer types, such as colorectal cancer, lung cancer, breast cancer, pancreatic cancer, and Ewing's sarcoma, and to be involved in abnormal growth, invasion, and metastasis [1,2,16,21,24,29]. However, the expression pattern (increase or decrease) of APLP2 is found inconsistent from cancer to cancer, though the consequences are not really clear [22]. And also, the role of APLP2 in glioblastoma still has not been elucidated.

In this study, we first searched a public tumor database Project Betastasis to learn APLP2 expression and its relationship with prognosis in glioblastoma. Further, we applied tissue microarray comprising a large number of glioma and normal brain tissue samples to clarify the expression of APLP2 and its prognostic significance in glioblastoma. Our results showed that there was no change of APLP2 expression in glioblastoma tissues compared to the normal ones, but a poor prognosis of glioblastoma patients was associated with a high APLP2 expression.

\section{Material and methods \\ Patients and tissue samples}

The study protocol and acquisition of tissue samples were approved by the Specialty Committee on Ethics of Biomedical Research, Second Military Medical University. Glioma tissue samples were obtained from glioma patients who underwent surgery at Changzheng Hospital between 1999 and 2010. Nor- mal brain tissue samples were obtained from trauma patients with severe head injuries, on whom partial resection of the brain was urgently needed. All the patients and legal guardians signed the informed consent forms of human tissue acquisition and used in this study following the National Regulations on the Use of Clinical Samples in China.

\section{Tissue microarray and immunohistochemistry}

The construction of microarray and immunohistochemistry were performed in Shanghai Biochip Company, Ltd as described previously [3,12]. A polyclonal anti-APLP2 antibody (1 : 200; Abcam, Cambridge, MA, USA) was used for immunohistochemistry staining according to the manufacturer's instructions. All sections were scored by the intensity and extent of APLP2 expression by two pathologists independently. In particular, the intensity scored 0 to 3 (0: negative; 1: light brown; 2: medium brown; 3: dark brown), and the percentage of positive cells was scored as its value. The two scores of intensity and positive percentage were multiplied to estimate the expression level of APLP2.

\section{Statistical analysis}

All statistical analyses were performed with the SPSS 18.0 software (Chicago, IL, USA). Each experiment with means and standard deviation (SD) was subjected to student $t$-test. Wilcoxon rank-sum test was used to compare the difference of counting data between two groups. OS and PFS curves were plotted by the Kaplan-Meier method and compared by log-rank test. The identification of relevant prognostic factors was performed by univariate analysis, multivariate analysis, and stepwise backward Cox regression model. Variables with a value of $p \leq 0.2$ on univariate analysis were added into the multivariate analysis. All statistical tests were two-tailed, the results were considered statistically significant when the $p$ values were less than 0.05 .

\section{In silico analysis}

The Project Betastasis (http://www.betastasis. com) provides mRNA expression and survival data of hundreds of glioma cases from two publically available datasets: Repository for Molecular Brain Neoplasia Data (REMBRANDT) and TCGA. The APLP2 expression data (Affymetrix HG U133 v2.0 Plus and 
Table I. Demographic and clinicopathological characteristics of glioblastoma patients

\begin{tabular}{|c|c|c|c|}
\hline \multirow[t]{2}{*}{ Characteristics } & \multirow[t]{2}{*}{ Value } & \multicolumn{2}{|c|}{ APLP2 expression } \\
\hline & & Low & High \\
\hline \multicolumn{4}{|l|}{ Age (years) } \\
\hline$<60$ & $85(74.6 \%)$ & 41 & 44 \\
\hline$\geq 60$ & $29(25.4 \%)$ & 10 & 19 \\
\hline \multicolumn{4}{|l|}{ Gender } \\
\hline Male & $74(64.9 \%)$ & 33 & 41 \\
\hline Female & $40(35.1 \%)$ & 18 & 22 \\
\hline \multicolumn{4}{|l|}{ Seizure } \\
\hline Yes & 14 (12.3\%) & 6 & 8 \\
\hline No & $100(87.7 \%)$ & 45 & 55 \\
\hline \multicolumn{4}{|l|}{ IICP } \\
\hline Yes & $50(43.9 \%)$ & 22 & 28 \\
\hline No & $64(56.1 \%)$ & 29 & 35 \\
\hline \multicolumn{4}{|l|}{ Cystic degeneration } \\
\hline Yes & 29 (25.4\%) & 20 & 9 \\
\hline No & $85(74.6 \%)$ & 31 & 54 \\
\hline \multicolumn{4}{|l|}{ Necrosis on MRI } \\
\hline Yes & $20(17.5 \%)$ & 12 & 8 \\
\hline No & $94(82.5 \%)$ & 39 & 55 \\
\hline \multicolumn{4}{|l|}{$\operatorname{MTD}(\mathrm{cm})$} \\
\hline$<5$ & $48(42.1 \%)$ & 24 & 24 \\
\hline$\geq 5$ & $66(57.9 \%)$ & 27 & 39 \\
\hline \multicolumn{4}{|l|}{ Primary/Secondary } \\
\hline Primary & $101(88.6 \%)$ & 45 & 56 \\
\hline Secondary & $13(11.4 \%)$ & 6 & 7 \\
\hline \multicolumn{4}{|l|}{ Resection degree } \\
\hline Gross total resection & $89(78.1 \%)$ & 39 & 50 \\
\hline Subtotal resection & $22(19.3 \%)$ & 11 & 11 \\
\hline Partial resection & $3(2.6 \%)$ & 1 & 2 \\
\hline \multicolumn{4}{|l|}{ Chemotherapy } \\
\hline Yes & $30(26.3 \%)$ & 16 & 14 \\
\hline No & 84 (73.7\%) & 35 & 49 \\
\hline \multicolumn{4}{|l|}{ Radiotherapy } \\
\hline Yes & 82 (71.9\%) & 36 & 46 \\
\hline No & 32 (28.1\%) & 15 & 17 \\
\hline \multicolumn{4}{|l|}{ Survival status } \\
\hline Live & $6(5.3 \%)$ & 4 & 2 \\
\hline Dead & 104 (91.2\%) & 44 & 60 \\
\hline Unknown & $4(3.5 \%)$ & 3 & 1 \\
\hline \multicolumn{4}{|l|}{ Recurrence } \\
\hline No & $4(3.5 \%)$ & 2 & 2 \\
\hline Yes & $106(93.0 \%)$ & 46 & 60 \\
\hline Unknown & $4(3.5 \%)$ & 3 & 1 \\
\hline
\end{tabular}

Affy Human Exon 1.0 ST generated by REMBRANDT and TCGA, respectively) were collected, and a Student's $t$ test was used to estimate expressional differences between glioblastomas and normal brain samples. In Kaplan-Meier, the median expression value of APLP2 was used as a cut-off value in both REMBRANDT and TCGA glioblastoma groups. Logrank $p$ value was calculated in Project Betastasis.

\section{Results \\ General clinical characteristics of glioma patients}

A total of 269 glioma tissue samples (9 Grade I, 101 Grade II, 45 Grade III, and 114 Grade IV) and 16 normal brain tissue samples were eligible in our study for further evaluation. The period of follow-up of 114 glioblastoma patients was from 0.5 to 102 months. The recurrence was found in 106 (93\%) cases, and only 6 (5.3\%) patients were still alive. The median OS and median PFS were 11 months (95\% Cl: $9.460-12.540)$ and 9.5 months $(95 \% \mathrm{Cl}$ : $7.976-$ 12.024), respectively. The clinicopathological characteristics of patients with glioblastoma are listed in Table I.

\section{Different changes of the APLP2 expression in glioblastoma at mRNA and protein levels}

To determine the expression level of the APLP2 in glioblastoma, we first analyzed the transcriptional alterations of APLP2 in Project Betastasis from REMBRANDT (214 glioblastoma samples) and TCGA (201 glioblastoma samples) cohorts. APLP2 mRNA expression data of twenty-one and eleven normal brains were also collected from the two datasets, respectively. It was shown that no transcriptional change of APLP2 was detected in the REMBRANDT glioblastoma cohort compared to normal brains $(2848.063 \pm 663.896$ vs. $2798.561 \pm 427.910, p=0.738)$ (Fig. 1A). Additionally, when calculating in astrocytoma (66 samples) and oligodendroglioma (145 samples) groups with an unknown pathological grade from REMBRANDT, our results found a statistical difference of APLP2 mRNA expression between astrocytoma and the normal control $(2508.292 \pm 555.148$ vs. $2798.561 \pm 427.910, p=0.023)$ (Fig. 1A). Moreover, the difference of APLP2 mRNA expression between glioblastoma samples and normal brain tissues from TCGAdataset has no statistical significance (2325.871 

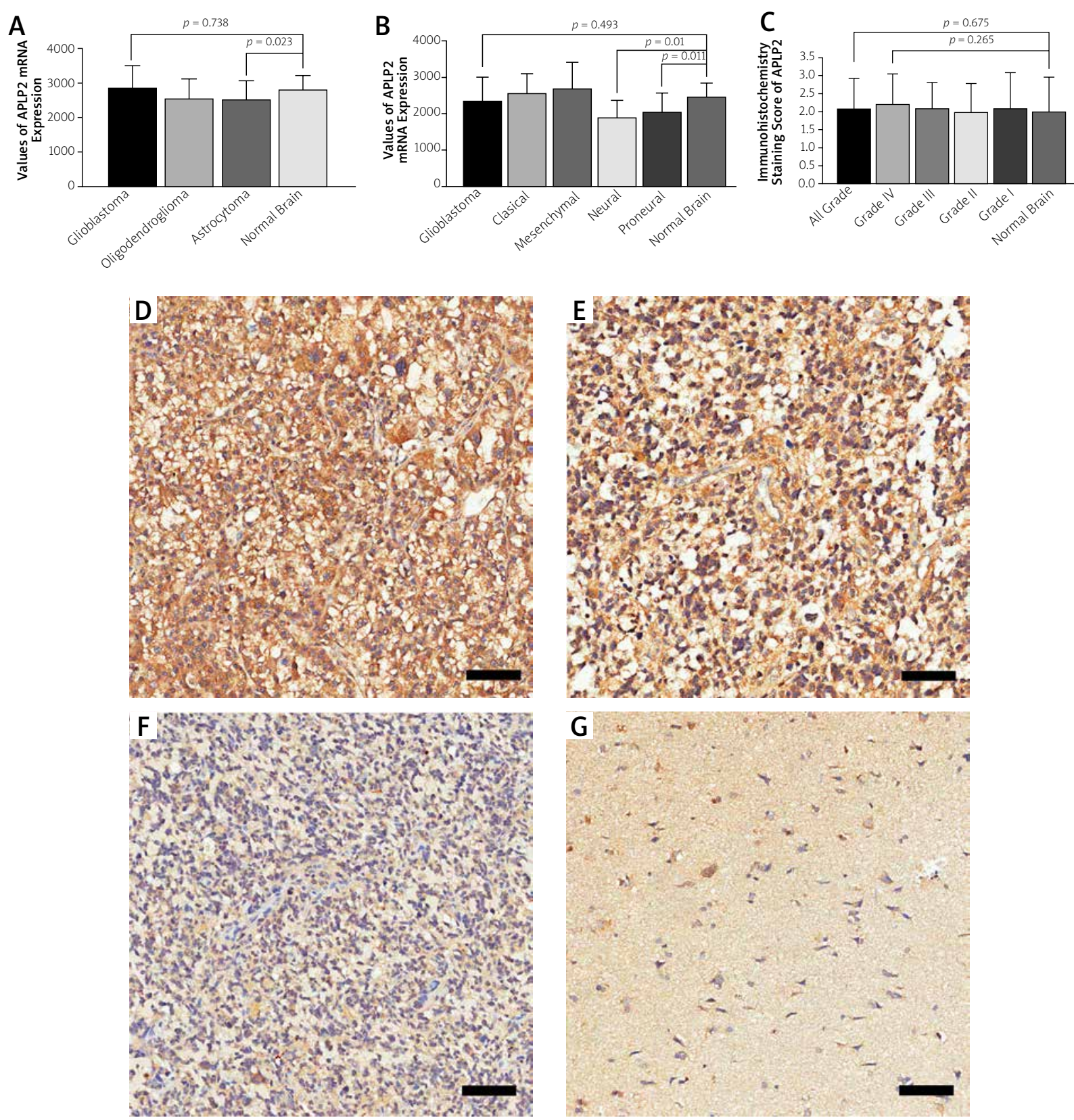

Fig. 1. APLP2 expression shows no significant difference between glioblastoma and normal brain. A) The level of APLP2 mRNA detected in glioblastoma $(n=214)$ from REMBRANDT dataset is statistically similar to normal brain $(n=21)(p=0.738)$, but a slight decrease of APLP2 mRNA is found in astrocytoma with unknown grade $(n=66)$ compared to that in the control group $(p=0.023)$. B) The APLP2 mRNA expression also has no obvious change in all glioblastoma cases $(n=201)$ and normal brain $(n=11)$ from TCGA dataset, but is moderately down-regulated in two subgroups of glioblastoma (Neural, $n=33, p=0.01$; Proneural, $n=57$, $p=0.011)$. C) Compared to normal brain $(n=16)$, the expression of APLP2 protein is insignificantly changed in patients with glioblastoma $(n=114)$ from our cohort $(p=0.265)$, and unrelated to pathological grade $(p>0.05)$. D-G) Representative staining images in glioblastoma samples and normal brain are shown in D-F and G, respectively. Most APLP2 protein expresses in the cytoplasm of glioblastoma cells (200× magnification). 
\pm 679.481 vs. $2467.725 \pm 363.005, p=0.493$ ) (Fig. 1 B) However, TCGA dataset analysis indicated that two subtypes of glioblastoma (33 Neural and 57 Proneural) expressed decreased APLP2 mRNA compared to the normal brain $(1880.072 \pm 485.411$ vs. $2467.725 \pm$ $363.005, p=0.01 ; 2030.734 \pm 531.831$ vs. $2467.725 \pm$ $363.005, p=0.011$, respectively), although Classical (53 samples) and Mesenchymal (58 samples) subtype groups did not (Fig. 1B).

We further validated APLP2 protein expression in our glioblastoma cohort (114 samples) by immunohistochemistry staining. Consistently, compared to normal brain tissues, the expression of APLP2 protein was insignificantly changed in patients with glioblastoma $(2.204 \pm 0.848$ vs. $1.997 \pm 0.965$, $p=0.265$ ) (Fig. 1C). In addition, the expression of APLP2 protein was assessed in different grades of glioma. As shown in Figure 1C-G, undifferentiated APLP2 protein expression was found among Grade I-IV groups and normal brains.

\section{APLP2 expression predicts survival time and serves as an independent prognostic factor for glioblastoma patients}

The relationship between the APLP2 expression level and clinical outcome was preliminarily examined in Project Betastasis. For the REMBRANDT glioblastoma cohort (178 cases), survival analysis showed that patients with a high expression of APLP2 had a shorter OS (376 days vs. 474 days, $p=0.0323$ ) (Fig. 2A). Though no statistically significant result was obtained in the OS analysis for the TCGA glioblastoma group (348 cases), there was still a moderate difference between high and low APLP2 subgroups (418 days vs. 447 days, $p=0.0578$ ) (Fig. 2B). Furthermore, the association between APLP2 expression and prognosis of glioblastoma from each subtype group in TCGA dataset was investigated. Notably, glioblastoma patients with a high APLP2 expression had a significantly worse outcome than low APLP2 expression ones in the proneural group (57 cases) (297 days vs. 748 days, $p=0.00373$ ) (Fig. 2C).

In our glioblastoma cohort, the median APLP2 expression score 2.70 was used as the cutoff value for survival analysis and divided patients into High $(\geq 2.70)$ and Low (< 2.70) groups. As determined by the log-rank test, a high APLP2 expression was identified as a risk factor for OS (10 months vs. 12 months, $p=0.029$ ) and PFS ( 8 months vs. 12 months, $p=0.018$ ) of glioblastoma patients (Fig. 2D-E and Table II). Meanwhile, among other clinical features, age less than 60 years and a high resection degree were found to be protective factors for OS, as well as age less than 60 years for PFS (Table II). Variables with $p \leq 0.2$ in the above univariate analysis were then added into Cox proportional hazards regression model to estimate the ability of APLP2 to predict survival. As a result, a high expression of APLP2 $(H R=1.537, p=0.041)$ and small extent of resection were demonstrated as independent risk factors for shorter OS in glioblastoma patients (Table III). As for PFS of glioblastoma patients, the multivariate survival analysis indicated that a high APLP2 expression was also a risk factor $(\mathrm{HR}=1.783, p=0.037)$, and age less than 60 years, female, and radiotherapy acted as protective factors (Table III).

\section{Discussion}

Previous studies have shown that human tumors express APLP2 in various amounts [22]. An increased expression of APLP2 has been found in colon cancer, pancreatic cancer, and Ewing's sarcoma, and that its pro-cancer functions, including proliferation, migration, invasion, metastasis and immune escape, are becoming increasingly clear $[19,21,23,25]$. However, APLP2 is downregulated in lung neuroendocrine tumor, though the cause and effect are not well understood [2]. In the current study, we found no statistically significant difference between glioblastoma and normal brain in the level of APLP2 expression, which was also not related to the pathological grade of glioma but associated with the prognosis of glioblastoma patients.

Given the oncogenic role of APLP2 in diverse types of cancer [22], it is suggested that APLP2 may also promote the development of glioblastoma. Nevertheless, it is currently unknown how APLP2 is regulated and functions in this malignant tumor. A few studies have demonstrated that miRNA-153 can modulate APLP2 expression at the post-transcriptional level $[15,18]$. In glioblastoma, miRNA-153 shows a significantly reduced expression and works as a suppressor to decrease cancer cell proliferation and increase apoptosis $[4,8,34,35]$. Previously it has been shown that APLP2 is also subject to regulation by retinoic acid (RA), which can induce an increased expression of the gene [5]. Glioblastoma exhibits a unique aberrant expression profile of RA signaling 
A
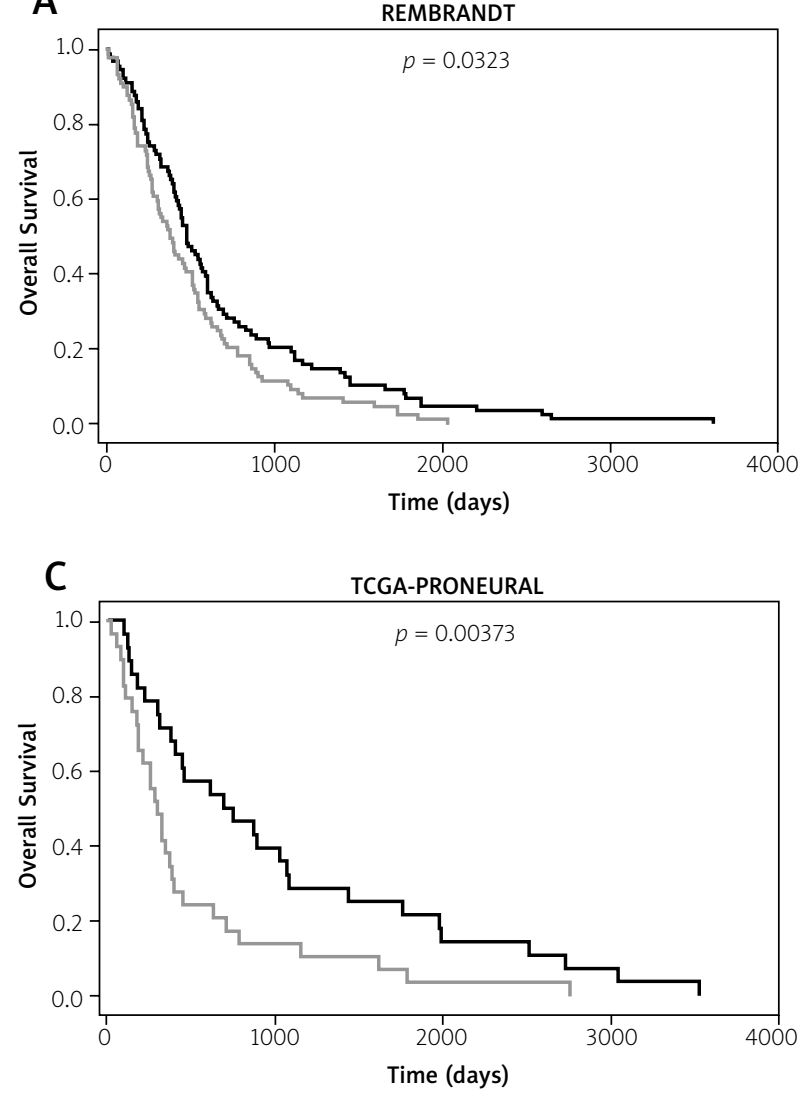

B

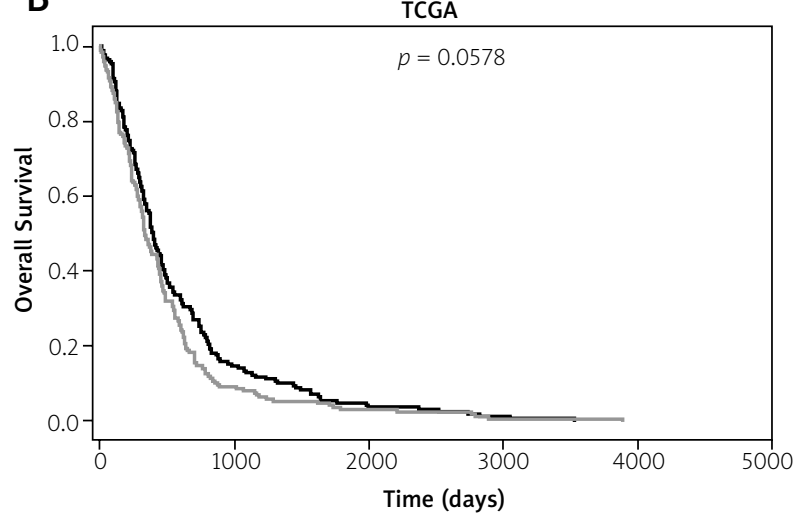

D

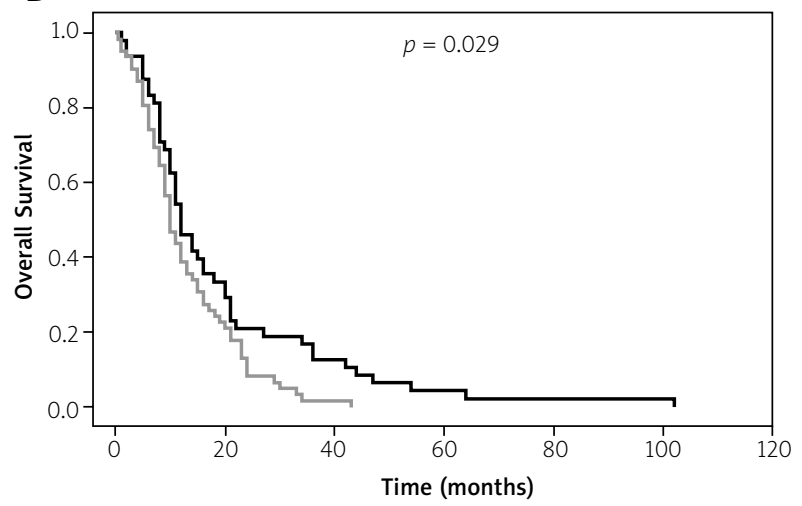

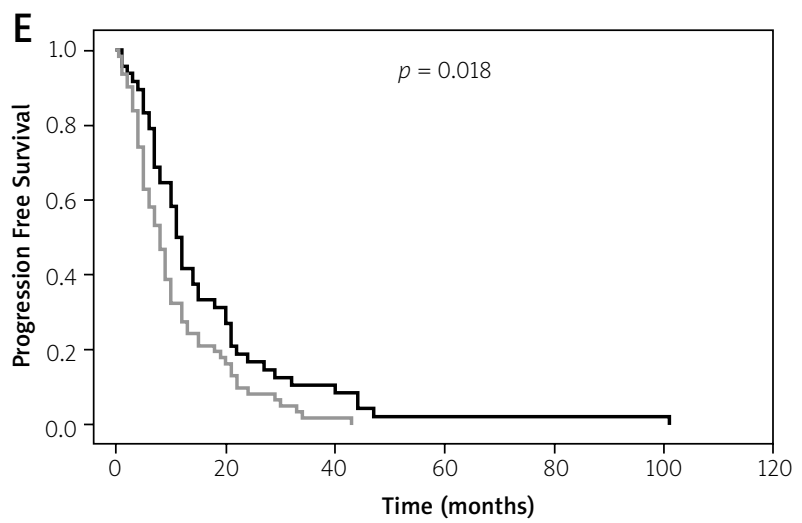

- APLP2 low

- APLP2 high

Fig. 2. Expression of APLP2 in glioblastoma is associated with patients' prognosis. A) Kaplan-Meier survival analysis in 178 glioblastoma cases from REMBRANDT stratified by APLP2 expression shows that a high expression of APLP2 predicts a short survival time (376 days vs. 474 days, $p=0.0323$ ). B) A moderate difference without statistical significance is obtained in OS analysis between high and low APLP2 subgroups from TCGA glioblastoma ( $n=348$ ) (418 days vs. 447 days, $p=0.0578)$. C) However, in the Proneural subgroup $(n=57)$, a patient with a high APLP2 expression has a significantly worse outcome than low APLP2 expression ones (297 days vs. 748 days, $p=0.00373$ ). D-E) Survival analysis in our glioblastoma cases $(n=114)$ stratified by APLP2 expression indicates both shorter OS (10 vs. 12 months, $p=0.029)$ and PFS (8 vs. 12 months, $p=0.018$ ) in patients with a higher APLP2 expression. 
Table II. Univariate analysis of factors associated with survival and progression of glioblastoma patients

\begin{tabular}{|lcccccc|}
\hline & \multicolumn{3}{c}{ OS } & \multicolumn{2}{c|}{ PFS } \\
\cline { 2 - 7 } & HR & $95 \% \mathrm{Cl}$ & $p$ & $\mathrm{HR}$ & $95 \% \mathrm{Cl}$ & $p$ \\
\hline Age $(\geq 60$ vs. $<60$ y) & 1.634 & $1.047-2.549$ & 0.031 & 1.712 & $1.098-2.669$ & 0.018 \\
\hline Gender (female vs. male) & 0.847 & $0.561-1.278$ & 0.428 & 0.741 & $0.492-1.115$ & 0.150 \\
\hline Seizure (yes vs. no) & 0.777 & $0.424-1.423$ & 0.414 & 0.999 & $0.557-1.793$ & 0.998 \\
\hline IICP (yes vs. no) & 1.024 & $0.695-1.510$ & 0.904 & 0.942 & $0.640-1.385$ & 0.761 \\
\hline MTD $(\geq 5$ vs. $<5$ cm) & 1.004 & $0.678-1.488$ & 0.984 & 1.264 & $0.854-1.870$ & 0.242 \\
\hline Cystic Degeneration (yes vs. no) & 0.637 & $0.401-1.012$ & 0.056 & 0.698 & $0.447-1.089$ & 0.113 \\
\hline Necrosis (yes vs. no) & 1.022 & $0.597-1.747$ & 0.938 & 1.401 & $0.841-2.333$ & 0.196 \\
\hline Primary (vs. secondary) & 0.807 & $0.441-1.476$ & 0.468 & 1.177 & $0.655-2.113$ & 0.586 \\
\hline APLP2 (high vs. low) & 1.540 & $1.027-2.311$ & 0.037 & 1.578 & $1.063-2.344$ & 0.024 \\
\hline Resection degree & & & 0.031 & & & 0.084 \\
\hline Total vs. partial & 0.217 & $0.067-0.703$ & 0.011 & 0.285 & $0.089-0.918$ & 0.035 \\
\hline Subtotal vs. partial & 0.193 & $0.056-0.664$ & 0.009 & 0.250 & $0.073-0.850$ & 0.026 \\
\hline Chemotherapy (yes vs. no) & 0.669 & $0.432-1.036$ & 0.072 & 0.800 & $0.517-1.237$ & 0.315 \\
\hline Radiotherapy (yes vs. no) & 0.666 & $0.436-1.017$ & 0.060 & 0.715 & $0.469-1.089$ & 0.118 \\
\hline
\end{tabular}

IICP - increased intracranial pressure, MTD - mean tumor diameter, HR - hazard ratio, OS - overall survival, PFS - progression-free survival

Table III. Multivariate analysis of factors associated with survival and progression of glioblastoma patients

\begin{tabular}{|c|c|c|c|c|c|}
\hline Survival* $^{\star}$ & Median surviva & nths, $95 \% \mathrm{Cl}$ ) & $p$ & HR & $95 \% \mathrm{Cl}$ \\
\hline \multicolumn{6}{|l|}{ OS } \\
\hline Age ( $\geq 60$ vs. $<60$ y) & & & 0.063 & & \\
\hline Cystic degeneration (yes vs. no) & & & 0.304 & & \\
\hline APLP2 (high vs. low) & $10(8.117-11.883)$ & $12(8.692-15.308)$ & 0.041 & 1.537 & $1.017-2.322$ \\
\hline Resection degree & & & 0.031 & & \\
\hline Total vs. partial & $11(9.447-12.553)$ & $2(0.400-3.600)$ & 0.010 & 0.208 & $0.063-0.687$ \\
\hline Subtotal vs. partial & $13(8.403-17.597)$ & $2(0.400-3.600)$ & 0.010 & 0.193 & $0.055-0.671$ \\
\hline Chemotherapy (yes vs. no) & & & 0.411 & & \\
\hline Radiotherapy (yes vs. no) & & & 0.076 & & \\
\hline \multicolumn{6}{|l|}{ PFS } \\
\hline Age ( $\geq 60$ vs. $<60$ y) & $6(4.534-7.466)$ & $11(9.282-12.718)$ & 0.014 & 1.788 & $1.126-2.841$ \\
\hline Gender (female vs. male) & $11(7.142-14.858)$ & $9(6.608-11.392)$ & 0.037 & 0.602 & $0.374-0.969$ \\
\hline Necrosis (yes vs. no) & & & 0.086 & & \\
\hline Cystic degeneration (yes vs. no) & & & 0.252 & & \\
\hline APLP2 (high vs. low) & $8(5.900-10.100)$ & $12(10.362-13.638)$ & 0.007 & 1.783 & $1.171-2.715$ \\
\hline Resection degree & & & 0.113 & & \\
\hline Total vs. partial & & & 0.781 & & \\
\hline Subtotal vs. partial & & & 0.455 & & \\
\hline Radiotherapy (yes vs. no) & $10(8.075-11.925)$ & $8(6.156-9.844)$ & 0.029 & 0.589 & $0.367-0.946$ \\
\hline
\end{tabular}

HR - hazard ratio, OS - overall survival, PFS - progression-free survival

*Variables were adopted for their prognostic significance by univariate analysis $(p \leq 0.2)$

genes with an augmented intratumoral RA level and alternative, pro-survival RA usage [6,27]. However, both decreased miRNA-153 and elevated RA levels could not increase APLP2 expression in glioblastoma, and other unknown but predominant mechanisms involved in the process need further investigations. 
Current studies have shown evidence that APLP2 plays a key role in the essential biological decision of differentiating a neuronal stem cell into a neuron [28]. Recent findings also indicate that these normal stem cells have the similar phenotype and function with glioblastoma stem cells, including self-renewal and multi-lineage differentiation [33]. It is revealed that both types of stem cell may share some common molecular mechanisms such as the APLP2 signal. APLP2 is a trans-membrane protein, the intracellular domain of which contains a YENPTY motif and can bind several adapter molecules some of which are involved in the control of neurogenesis such as Dab1 and Numb $[14,26]$. Numb is involved in cell fate decisions such as proliferation versus differentiation by its repressing activity on Notch $[26,28]$. Consistently, Numb shows a similar expression pattern as APLP2 in glioblastoma (http://www.betastasis.com). Moreover, Numb also has been found to specify glioblastoma stem cell fate, although this manner does not require Notch inhibition [11]. Thus, there is an urgent need to discover the possible role of APLP2 in this cancer stem cell, which may explain the unchanged expression between glioblastoma tissues and normal brains.

Conclusively, we demonstrate that APLP2 expression is not significantly different among glioblastoma and normal brain, however, a high level of APLP2 is associated with poor prognosis in the glioblastoma patient group and APLP2 can work as an independent risk factor for OS. Additionally, understanding the function and molecular pathways involved by APLP2 will facilitate the development of therapeutic strategies for glioblastoma.

\section{Acknowledgements}

This work was supported by the National Natural Science Foundation (81572501 and 81272781), Program for Academic Leaders of Shanghai (No. 043), and "Pu Jiang Talent" Project of Shanghai (PJ[2014]0002617).

\section{Disclosure}

The authors report no conflict of interest.

\section{References}

1. Abba MC, Drake JA, Hawkins KA, Hu Y, Sun H, Notcovich C, Gaddis S, Sahin A, Baggerly K, Aldaz CM. Transcriptomic changes in human breast cancer progression as determined by serial analysis of gene expression. Breast Cancer Res 2004; 6: R499-513.

2. Arvidsson Y, Andersson E, Bergstrom A, Andersson MK, Altiparmak G, Illerskog AC, Ahlman H, Lamazhapova D, Nilsson O. Amyloid precursor-like protein 1 is differentially upregulated in neuroendocrine tumours of the gastrointestinal tract. Endocr Relat Cancer 2008; 15: 569-581.

3. Bachoo RM, Maher EA, Ligon KL, Sharpless NE, Chan SS, You MJ, Tang Y, DeFrances J, Stover E, Weissleder R, Rowitch DH, Louis DN, DePinho RA. Epidermal growth factor receptor and Ink4a/Arf: convergent mechanisms governing terminal differentiation and transformation along the neural stem cell to astrocyte axis. Cancer Cell 2002; 1: 269-277.

4. Barciszewska AM. MicroRNAs as efficient biomarkers in highgrade gliomas. Folia Neuropathol 2016; 54: 351-359.

5. Beckman M, Iverfeldt K. Increased gene expression of beta-amyloid precursor protein and its homologues APLP1 and APLP2 in human neuroblastoma cells in response to retinoic acid. Neurosci Lett 1997; 221: 73-76.

6. Campos B, Centner FS, Bermejo JL, Ali R, Dorsch K, Wan F, Felsberg J, Ahmadi R, Grabe N, Reifenberger G, Unterberg A, Burhenne J, Herold-Mende C. Aberrant expression of retinoic acid signaling molecules influences patient survival in astrocytic gliomas. Am J Pathol 2011; 178: 1953-1964.

7. Cancer Genome Atlas Research N. Comprehensive genomic characterization defines human glioblastoma genes and core pathways. Nature 2008; 455: 1061-1068.

8. Cui Y, Zhao J, Yi L, Jiang Y. microRNA-153 Targets mTORC2 Component Rictor to Inhibit Glioma Cells. PLoS One 2016; 11: e0156915.

9. Guo J, Thinakaran G, Guo Y, Sisodia SS, Yu FX. A role for amyloid precursor-like protein 2 in corneal epithelial wound healing. Invest Ophthalmol Vis Sci 1998; 39: 292-300.

10. Jacobsen KT, Iverfeldt K. Amyloid precursor protein and its homologues: a family of proteolysis-dependent receptors. Cell Mol Life Sci 2009; 66: 2299-2318.

11. Jiang X, Xing H, Kim TM, Jung Y, Huang W, Yang HW, Song S, Park PJ, Carroll RS, Johnson MD. Numb regulates glioma stem cell fate and growth by altering epidermal growth factor receptor and Skp1-Cullin-F-box ubiquitin ligase activity. Stem Cells 2012; 30: 1313-1326.

12. Kononen J, Bubendorf L, Kallioniemi A, Barlund M, Schraml P, Leighton S, Torhorst J, Mihatsch MJ, Sauter G, Kallioniemi OP. Tissue microarrays for high-throughput molecular profiling of tumor specimens. Nat Med 1998; 4: 844-847.

13. Korte M, Herrmann U, Zhang X, Draguhn A. The role of APP and APLP for synaptic transmission, plasticity, and network function: lessons from genetic mouse models. Exp Brain Res 2012; 217: 435-440.

14. Lakoma J, Garcia-Alonso L, Luque JM. Reelin sets the pace of neocortical neurogenesis. Development 2011; 138: 5223-5234.

15. Liang C, Zhu H, Xu Y, Huang L, Ma C, Deng W, Liu Y, Qin C. MicroRNA-153 negatively regulates the expression of amyloid precursor protein and amyloid precursor-like protein 2. Brain Res 2012; 1455: 103-113.

16. Li C, Kato M, Shiue L, Shively JE, Ares M, Jr, Lin RJ. Cell type and culture condition-dependent alternative splicing in human 
breast cancer cells revealed by splicing-sensitive microarrays. Cancer Res 2006; 66: 1990-1999.

17. Li XF, Thinakaran G, Sisodia SS, Yu FS. Amyloid precursor-like protein 2 promotes cell migration toward fibronectin and collagen IV. J Biol Chem 1999; 274: 27249-27256.

18. Long JM, Ray B, Lahiri DK. MicroRNA-153 physiologically inhibits expression of amyloid-beta precursor protein in cultured human fetal brain cells and is dysregulated in a subset of Alzheimer disease patients. J Biol Chem 2012; 287: 31298-31310.

19. Moss AC, Doran PP, Macmathuna P. In Silico Promoter Analysis can Predict Genes of Functional Relevance in Cell Proliferation: Validation in a Colon Cancer Model. Transl Oncogenomics 2007; 2: 1-16

20. Omuro A, DeAngelis LM. Glioblastoma and other malignant gliomas: a clinical review. JAMA 2013; 310: 1842-1850.

21. Pandey P, Rachagani S, Das S, Seshacharyulu P, Sheinin Y, Naslavsky N, Pan Z, Smith BL, Peters HL, Radhakrishnan P, McKenna NR, Giridharan SS, Haridas D, Kaur S, Hollingsworth MA, MacDonald RG, Meza JL, Caplan S, Batra SK, Solheim JC. Amyloid precursor-like protein 2 (APLP2) affects the actin cytoskeleton and increases pancreatic cancer growth and metastasis. Oncotarget 2015; 6: 2064-2075.

22. Pandey P, Sliker B, Peters HL, Tuli A, Herskovitz J, Smits K, Purohit A, Singh RK, Dong J, Batra SK, Coulter DW, Solheim JC. Amyloid precursor protein and amyloid precursor-like protein 2 in cancer. Oncotarget 2016; 7: 19430-19444.

23. Peters HL, Tuli A, Wang X, Liu C, Pan Z, Ouellette MM, Hollingsworth MA, Macdonald RG, Solheim JC. Relevance of amyloid precursor-like protein $2 \mathrm{C}$-terminal fragments in pancreatic cancer cells. Int J Oncol 2012; 41: 1464-1474.

24. Peters HL, Yan Y, Nordgren TM, Cutucache CE, Joshi SS, Solheim JC. Amyloid precursor-like protein 2 suppresses irradiation-induced apoptosis in Ewing sarcoma cells and is elevated in immune-evasive Ewing sarcoma cells. Cancer Biol Ther 2013; 14: 752-760.

25. Peters HL, Yan Y, Solheim JC. APLP2 regulates the expression of MHC class I molecules on irradiated Ewing's sarcoma cells. Oncoimmunology 2013; 2: e26293.

26. Roncarati R, Sestan N, Scheinfeld MH, Berechid BE, Lopez PA, Meucci O, McGlade JC, Rakic P, D’Adamio L. The gamma-secretase-generated intracellular domain of beta-amyloid precursor protein binds Numb and inhibits Notch signaling. Proc Natl Acad Sci U S A 2002; 99: 7102-7107.

27. Schug TT, Berry DC, Shaw NS, Travis SN, Noy N. Opposing effects of retinoic acid on cell growth result from alternate activation of two different nuclear receptors. Cell 2007; 129: 723-733.

28. Shariati SA, Lau P, Hassan BA, Muller U, Dotti CG, De Strooper B, Gartner A. APLP2 regulates neuronal stem cell differentiation during cortical development. J Cell Sci 2013; 126: 1268-1277.

29. Takagi K, Ito S, Miyazaki T, Miki Y, Shibahara Y, Ishida T, Watanabe M, Inoue S, Sasano H, Suzuki T. Amyloid precursor protein in human breast cancer: an androgen-induced gene associated with cell proliferation. Cancer Sci 2013; 104: 1532-1538.

30. Tuli A, Sharma M, Mcllhaney MM, Talmadge JE, Naslavsky N, Caplan S, Solheim JC. Amyloid precursor-like protein 2 increases the endocytosis, instability, and turnover of the $\mathrm{H} 2-\mathrm{K}(\mathrm{d}) \mathrm{MHC}$ class I molecule. J Immunol 2008; 181: 1978-1987.
31. Van Meir EG, Hadjipanayis CG, Norden AD, Shu HK, Wen PY, Olson $J$ J. Exciting new advances in neuro-oncology: the avenue to a cure for malignant glioma. CA Cancer J Clin 2010; 60: 166-193.

32. Walsh DM, Minogue AM, Sala Frigerio C, Fadeeva JV, Wasco W, Selkoe DJ. The APP family of proteins: similarities and differences. Biochem Soc Trans 2007; 35: 416-420.

33. Wu Q, Wang X. Neuronal stem cells in the central nervous system and in human diseases. Protein Cell 2012; 3: 262-270.

34. Xu J, Liao X, Wong C. Downregulations of B-cell lymphoma 2 and myeloid cell leukemia sequence 1 by microRNA 153 induce apoptosis in a glioblastoma cell line DBTRG-05MG. Int J Cancer 2010; 126: 1029-1035.

35. Zhao S, Deng Y, Liu Y, Chen X, Yang G, Mu Y, Zhang D, Kang J, Wu Z. MicroRNA-153 is tumor suppressive in glioblastoma stem cells. Mol Biol Rep 2013; 40: 2789-2798. 